\title{
Effects of ovule and seed abortion on brood size and fruit costs in the leguminous shrub Caesalpinia gilliesii (Wall. ex Hook.) D. Dietr.
}

Ana Calviño ${ }^{1,2}$

Received: 20 March, 2013. Accepted: 14 October, 2013

\begin{abstract}
For several plant species, brood size results from the abortion of ovules and seeds. However, these processes have rarely been studied together in wild plants. In some of the leguminous species studied, seed abortion has been found to depend on pollen quality and on the position of the ovule or fruit. The direct consequence for the mother plant is that fruit costs increase as the seed:ovule ratio decreases. However, because ovule abortion occurs earlier than does seed abortion, the former can reduce the biomass invested per seed (i.e., fruit costs) more efficiently than does the latter. Here, the frequencies of aborted ovules and seeds were analyzed in relation to the type of pollination treatment (open pollination vs. hand cross-pollination) and ovule/fruit position within pods of the leguminous shrub Caesalpinia gilliesii. The influence of ovule and seed abortion on fruit costs was analyzed by comparing the pericarp mass per seed between fruits with different frequencies of aborted ovules and seeds. The rate of ovule abortion was similar between hand cross-pollinated and open-pollinated fruits but was higher than that of seed abortion in one- and two-seeded fruits, as well as in those at stylar positions and in distal fruits. Hand cross-pollination reduced seed abortion but did not increase the seed:ovule ratio. In addition, fruits that aborted ovules were found to be less costly than were those that aborted seeds. From the mother plant perspective, these results indicate that ovule abortion is a more efficient mechanism of reducing fruit costs than is seed abortion, because fertilization opportunities decrease with position, and show that brood size is significantly influenced by the fate of the ovule at the pre-zygotic stage.
\end{abstract}

Key words: fruit mass, seed mass, pre-zygotic abortion, position effects, seed:ovule ratio

\section{Introduction}

The final number of seeds within a fruit is the result of a sequential process that starts with an initial number of ovules in the ovary and ends with the successful development of fertilized ovules. In plants in which predation is not a critical factor for seed production, the upper limit for seed number per fruit is set by the number of ovules in the ovary, and the final seed:ovule ratio is mainly determined by fertilization success and seed abortion. Pollen limitation usually constrains the number of ovules that develop into seeds, reducing fertilization success. However, even after fertilization has been achieved, abortion of developing embryos can substantially reduce the number of seeds. Seed abortion, which occurs at different developmental stages within the fruit, has been shown to be non-random and based on the genotype of the embryo (Korbecka et al. 2002); however, lower seed:ovule ratios can also result from the abortion of unfertilized ovules within the fruit (hereafter, ovule abortion). Causes of ovule abortion are diverse and are well known in crop plants that frequently exhibit abortion of older ovules. For instance, variability in ovule longevity within the ovary reduces the period for successful pollination, decreasing fruit and seed set in several Prunus spp., kiwis and sweet cherries, among other species (Sanzol \& Herrero 2001; Alburquerque et al. 2002; Cuevas et al. 2009). Ovule longevity depends on several factors, such as temperature during the flowering period and nitrogen availability, and it has been shown to vary with culture conditions (Goldwin 1992). In wild plants, variation in the stage of ovule development at anthesis has mainly been observed in orchids (O'Neill 1997) and has also been noted for Narcissus (Sage et al. 1999) and even for gymnosperms (Said et al. 1991). More recently, differential ovule receptivity at flower anthesis has been shown to reduce the number of fertile ovules in several leguminous plants (Sengupta \& Tandon 2010). As in crop plants, unreceptive ovules have been shown to abort before fertilization in leguminous species, as studied by Sengupta and Tandon (2010).

Independently of whether seed:ovule ratios are influenced by ovule or seed abortion, differences in seed number per fruit alter the cost of the fruit for the mother plant. Hence, plants invest fewer resources per seed in multi-seeded fruits than in few-seeded fruits (Obeso 2004).

\footnotetext{
${ }^{1}$ Universidad Nacional de Córdoba, Instituto Multidisciplinario de Biología Vegetal, Córdoba, Argentina

${ }^{2}$ Author for correspondence: anacalv@imbiv.unc.edu.ar
} 
Accordingly, as a strategy to conserve resources, mother plants avoid the development of fruits with few seeds (Obeso 2004). However, plants can still exhibit positively skewed frequency distributions for seed number per fruit, so that many fruits are usually few-seeded in several species (Uma Shaanker et al. 1988). Because ovule abortion occurs at an earlier developmental stage than does seed abortion (i.e., before fertilization), the former is expected to reduce fruit costs to a greater extent than does the latter, allowing the plant to redirect the conserved resources at an earlier stage. In a seminal paper, Lloyd (1980) indicated that abortion of floral organs "should be made as early as possible to minimize the wastage in unrewarded expenditure". In other words, the earlier the brood size is defined, the more equitable will be the resource allocation per seed. This assumption, which can be summarized as "the earlier the better" hypothesis, makes the study of ovule abortion a key factor in understanding brood size patterns and fruit costs in plants. Nevertheless, the extent to which few-seeded fruits result from ovule or seed abortion is unclear, because it is difficult to distinguish between the two processes (O'Donnel \& Bawa 1993). Therefore, most conclusions about brood size are based only on seed abortion patterns. The effects of ovule and seed abortion on fruit costs have yet to be studied simultaneously. Leguminous plants, in which usually many ovules abort before fertilization (Wiens et al. 1989), provide a suitable study system to test the effect of early abortion on seed:ovule ratios and fruit costs.

Caesalpinia gilliesii (Wall. ex Hook.) D. Dietr. (Fabaceae) is a leguminous shrub that grows in arid and semi-arid regions of Argentina. Flowers are arranged in inflorescences with a sequential blooming pattern. Fruit set in this species rarely exceeds $12 \%$, increasing with the addition of cross pollen (Jausoro \& Galetto 2001). In C. gilliesii, unfertilized and fertilized ovules (hereafter, ovules and seeds) both abort during fruit development, thus reducing the final seed set (Calviño 2006). As in other leguminous fruits, aborted seeds can be easily quantified in C. gilliesii, because non-viable seeds are clearly distinguishable from viable ones and seed predation is negligible (Calviño 2006). In addition, unfertilized, aborted ovules remain attached to the fruit wall, so that a typical mature pod of C. gilliesii has aborted ovules, plus aborted and viable seeds. This allows us to count the initial number of ovules within the ovary and the number of aborted ovules, as well as the initial and final number of fertilized seeds per fruit. Here, these features were used in order to study the relative contribution of ovule and seed abortion to brood size and fruit costs in pods of C. gilliesii. In addition, because the ovules of leguminous plants are arranged linearly, from the stylar end to the peduncular end of the ovary, unequal resource distribution, coupled with an unidirectional pollen tube entrance within the pod, might contribute to the understanding of non-random abortion patterns observed in these species, including the effects that position has on seed abortion (Bawa \& Buckley 1989) and ovule abortion (Sengupta \& Tandon 2010). Accordingly, it is possible that the position of the ovule/seed within the ovary and of the fruit within the inflorescence both influence abortion patterns in C. gilliesii fruits. Therefore, both of those elements were considered here.

The specific aims of this work were to examine the relative contribution of ovule and seed abortion to brood size in fruits of Caesalpinia gilliesii; to determine whether ovule and seed abortion depend on cross pollination; to analyze the probability of ovule and seed abortion in fruits of C. gilliesii in relation to ovule/seed position within fruits and to fruit position within inflorescences; and to analyze the influence of ovule and seed abortion levels on fruit costs.

\section{Material and methods}

Mature pods of Caesalpinia gilliesii were collected from six sites, with 36 mother plants per site ( 24 fruits per plant; $\mathrm{N}=864$ fruits), and the position of the fruit within the inflorescence (basal, medium and distal) was recorded in the field. Fruits were stored in individual paper bags at room temperature until processed. Aborted ovules can be easily distinguished from aborted seeds by their smaller size (0.5-1 vs. 3-6 $\mathrm{mm}$ in length; Fig. 1). The complete sample was used in order to explore brood size patterns in C. gilliesii and to record the frequency per fruit of aborted ovules, aborted seeds and viable seeds.

The probability of aborted ovules, aborted seeds and viable seeds in each fruit was assessed in a subsample of 540 fruits ( 15 fruits per plant). The subsample was used in order to select fruits with only eight initial ovules in the ovary and to avoid differences in seed number due to variability in ovule number. In those fruits, the status (aborted ovule, aborted seed or viable seed) and the position of each ovule/seed within the fruit were recorded considering the stylar end of the ovary as position number one. However,

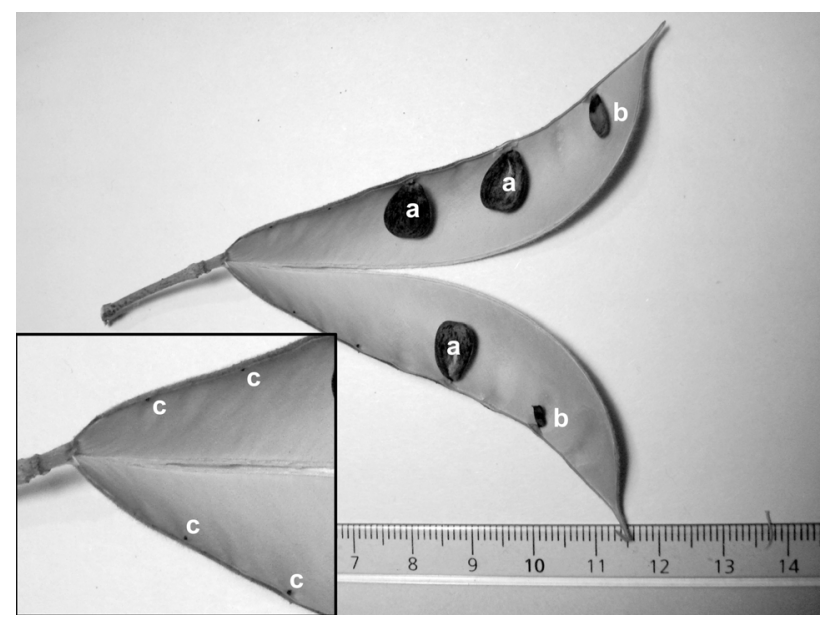

Figure 1. A typical Caesalpinia gilliesii fruit showing viable seeds (a), aborted seeds (b) and aborted ovules (c). 
the results of the analysis performed for the fruit sample as a whole (results not shown) did not differ significantly from those reported here.

To determine whether ovule and seed abortion depend on pollination, we hand cross-pollinated eight perfect flowers from 10 plants with a mix of fresh pollen from three neighboring plants at one site. Receptive stigmas were previously checked for the absence of a previous pollination event. At the end of the reproductive season, 57 fruits from cross-pollinated flowers were obtained (fruit set: $70 \%$ ), and the same number of open-pollinated fruits were collected from the same plants. All of those fruits were processed as described above.

To determine fruit cost, the mass of each fruit containing at least one seed and total seed mass per fruit were measured in a Mettler Toledo PB153 balance (Ohaus Corporation, Pine Brook, NJ, USA) at $0.0002 \mathrm{~g}(\mathrm{n}=470$ fruits). Fruit cost was then expressed as the pericarp mass invested per seed (PMS):

$\mathrm{PMS}=($ fruit mass - total seed mass $) /$ seed number per fruit

\section{Statistical analysis}

Statistical analyses were performed using $\mathrm{R}$ environment (R Development Core Team 2011). To address the relative contribution of ovule and seed abortion to brood size in Caesalpinia gilliesii fruits, we analyzed whether seed number per fruit and type of pollination treatment were associated with the frequency of aborted ovules or aborted seeds. Association patterns were explored with mosaic plots obtained with the strucplot framework (Meyer et al. 2006) and residual-based shading (Zeileis et al. 2007) of the $v c d$ package (Meyer et al. 2011). Mosaic plots are useful for visualizing association patterns of categorical data and consist of an area composed of rectangles (i.e., tiles), which correspond to the cell frequencies in a contingency table (Meyer et al. 2006). Each cell in the table is represented in the mosaic plot by a tile with height proportional to the residuals and width proportional to the square root of the expected frequencies (Meyer et al. 2006). In the shading_Friendly function used here, tiles with dashed lines correspond to observed frequencies that were lower than expected, whereas those with solid lines correspond to observed frequencies that were greater than expected, by tests of independence (Meyer et al. 2006). Only filled tiles of the plot indicate a significant association according to Pearson's chi-square test. Mosaic plots were also used in order to test the association between seed number and pollination treatment (open pollination vs. hand cross-pollination).

The probability of aborted ovules, aborted seeds and seed viability in relation to ovule/seed position within fruits and to fruit position within inflorescences was analyzed with a generalized linear mixed effects models (lmer function of the lme4 package, Bates et al. 2011) with aborted ovules and aborted seeds as binary variables. The spatial structure of the data was as follows: site $(n=6)$ as random effect, mother plant $(\mathrm{n}=36)$ as random effect nested within site, fruit position ( $\mathrm{n}=3$; basal, medium and distal) as fixed effect nested within plant and site, and ovule/seed position $(\mathrm{n}=8)$ as fixed effect nested within fruit position, mother plant and site. We analyzed the probability of success of a total of 4320 ovules and 2819 fertilized seeds of 540 fruits.

The influence that the rates of ovule and seed abortion had on the PMS was analyzed with a linear mixed effects model (lme function of the nlme package, Pinheiro et al. $2011)$ in fruits that set at least one seed $(n=470$ fruits from 16 mother plants and four sites). Aborted ovules and aborted seeds per fruit were negatively correlated within a fruit (results not shown). Therefore, a unique factor of relative ovule/seed abortion per fruit was used. The relative ovule abortion (ROA) rate was calculated as follows:

$\mathrm{ROA}=[$ aborted ovules $/($ aborted ovules + aborted seeds $)] \times 100$

Four levels of ROA were defined: ROA $=0 \%$ (for fruits with no aborted ovules but aborted seeds); $0 \%>$ ROA $\leq 50 \%$ (for fruits with more aborted seeds than aborted ovules or an equal number of both); $50 \%<\mathrm{ROA}<100 \%$ (for fruits with more aborted ovules than aborted seeds); and ROA = $100 \%$ (for fruits with aborted ovules only). Because lower-cost fruits might compensate for low PMS with a high seed number or seed mass, the influence of ROA on seed number and mean seed mass per fruit was also analyzed. If ovule abortion allowed plants to conserve resources, fruits with an ROA of $0 \%$ (i.e., those that aborted only seeds) would be expected to present higher costs and higher PMS than would fruits with an ROA $>0 \%$, although seed number and seed mass would be similar. In contrast, if fruits that aborted more ovules invested less biomass per seed (i.e., had a lower PMS) but also produced fewer seeds than fruits with lower abortion rates, ovule abortion per se would be interpreted as an inefficient mechanism of resource conservation, because ovule abortion could occur at the expense of seed number or seed mass per fruit. Therefore, to effectively address the influence of ovule abortion on fruit costs, it is important to consider variability in seed number and in seed mass. The effect of ROA on mean seed mass was analyzed with lme, whereas its effect on seed number was analyzed with lmer and Poisson distribution. In all cases, the level of ROA was considered a fixed effect, and mother plant nested within sites was considered a random effect. In lme models, the significance of fixed effects was determined with F-tests, and model parameters were estimated with restricted maximum likelihood, and in lmer models with Wald-z and Laplace approximation for fixed effects and model parameters, respectively. The effect of random terms was tested by fitting two nested models with and without each random term and comparing the two models with likelihood ratio tests (Zuur et al. 2009). 


\section{Results}

Fruits of Caesalpinia gilliesii had a median of eight initial ovules, of which a median of five remained fertile after ovule abortion (Fig. 2a). Fertilization success of these ovules was complete, $100 \%$ of the viable ovules becoming fertilized in a fruit (Fig. 2b). From these initial seeds, a median of three seeds per fruit reached maturity after seed abortion. Fifty percent of the fruits had one, two or three seeds, which yielded a positively skewed frequency distribution for seed number (Fig. 2b). Of the fruit sample as a whole, $12.64 \%$ of the fruits contained no viable seeds but did contain at least one aborted seed per fruit. These "seedless" fruits had a median of four aborted seeds per fruit, compared with a median of two aborted seeds in one- and two-seeded fruits. In the sequential process of seed development, $35 \%$ of the initial ovules in the ovary aborted before fertilization, $25.4 \%$ were fertilized but aborted later, and $39.56 \%$ succeeded in becoming a viable seed. Only 68 seeds were predated from the fruit sample as a whole $(<2 \%)$. Predated fruits and seeds were excluded from further analyses.
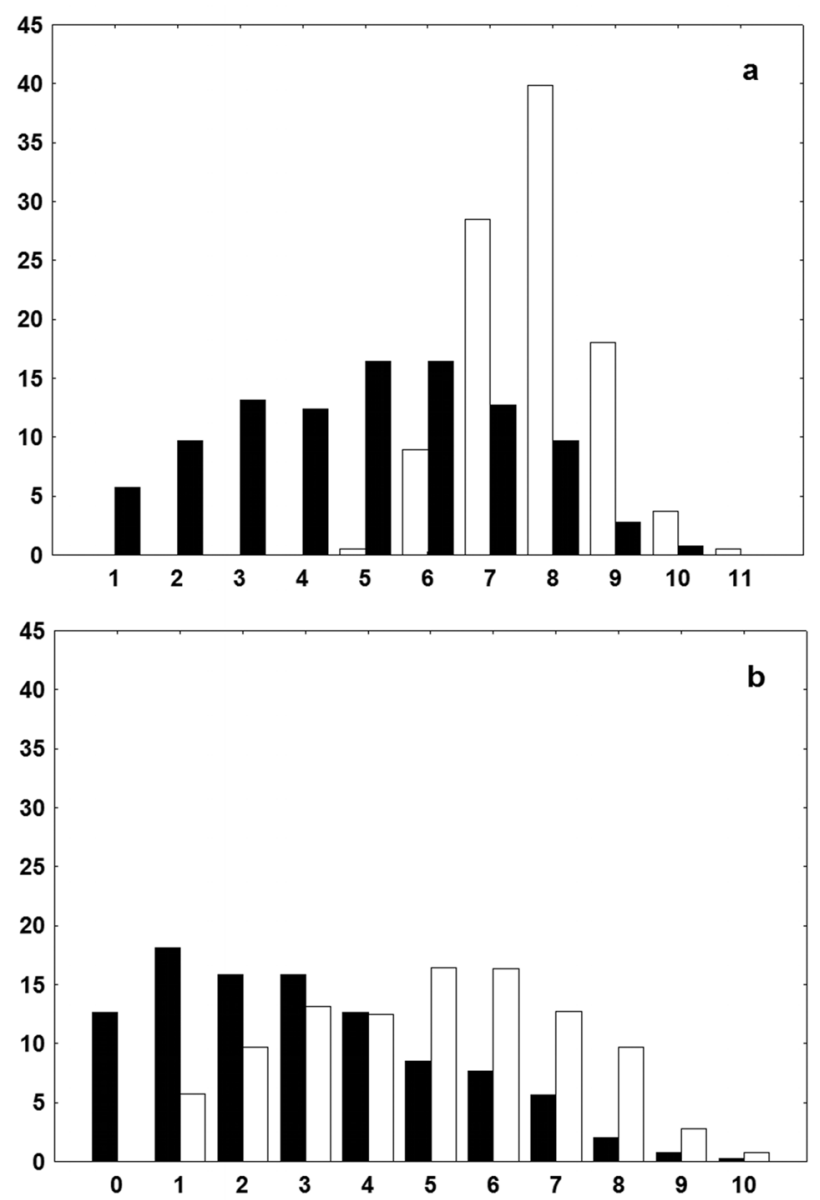

Figure 2. Relative frequency distributions for Caesalpinia gilliesii fruits $(\mathrm{N}=$ 864): in a, distributions for fruits with different numbers of ovules before (white bars), and after (black bars) ovule abortion; and, in b, distributions for seeds per fruit before (white bars), and after (black bars) seed abortion.

\section{Abortion levels, brood size and pollination treatment}

Abortion of ovules and seeds varied among fruits with different seed numbers (Fig. 3). In seedless fruits, the number of aborted seeds was higher than was that of aborted ovules, whereas that of aborted ovules was significantly higher than was that of aborted seeds in one- and two-seeded fruits (Fig. 3). Fruits with three or more seeds exhibited similar frequencies of aborted ovules and aborted seeds.

Ovule abortion was independent of the type of pollination treatment (Fig. 4a), the rate of ovule abortion being comparable between fruits obtained from hand crosspollination and those obtained from open-pollination ( $n=57$ for each treatment). In contrast, the frequency of aborted seeds per fruit was greater than expected in the open-pollinated fruits, which was not the case for the hand cross-pollinated fruits (Fig 4a). Nevertheless, the frequency of fruits with different seed numbers was independent of the type of pollination treatment (Fig. 4b).

\section{Influence of ovule/seed and fruit positions on abortion}

The position of the ovule within the ovary had a significant effect on the probability of abortion, with ovules at basal positions being more likely to abort than ovules at stylar positions $(z \geq 4.64, p<0.0001)$. The probability of ovule abortion increased significantly from position number 4 to position number 8 (Fig. 5). In contrast, seed abortion probabilities decreased from positions 5 to $8(z \geq-2.98, p \leq 0.003)$, with seeds proximate to the stylar end having the lowest probabilities of aborting (Fig. 5). Viable seeds were also located near the stylar end, and their probability of aborting decreased from positions 4 to 8 ( $z \geq-2.861, p \leq 0.004$, Fig. 5).

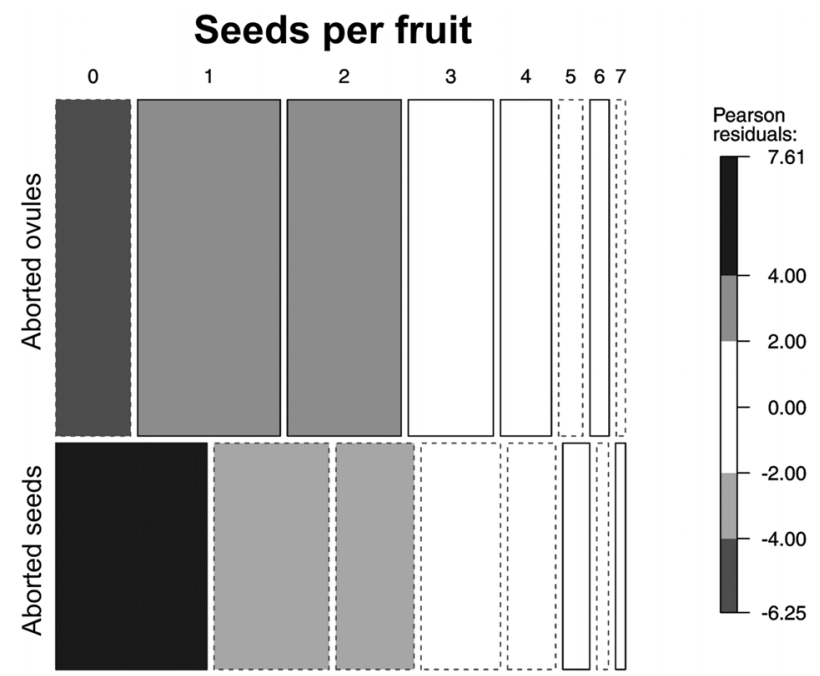

Figure 3. Mosaic plot for the frequency distribution of aborted ovules (aborted ovules) and seeds (aborted seeds) within Caesalpinia gilliesii fruits with different seed number. Dashed lines and solid lines indicate reductions and increases, respectively, in relation to the expected frequencies. Only filled tiles represent observed frequencies that were significantly different than expected according to Pearson's chi-square test ( $\mathrm{N}=864$ fruits). 

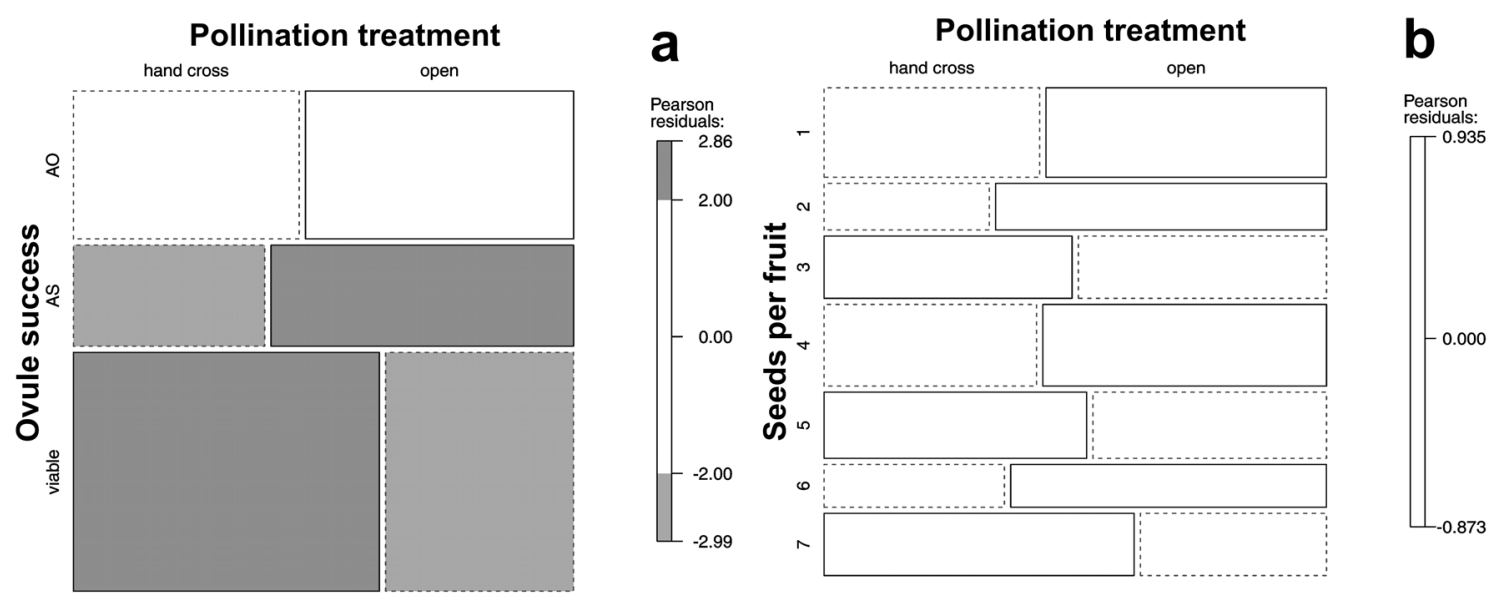

Figure 4. Mosaic plots for the frequency of viable seeds, aborted seeds (AS) and aborted ovules (AO) within fruits of Caesalpinia gilliesii (a), and the frequency of different seeded fruits in relation to the type of pollination treatment (b). Dashed lines and solid lines indicate reductions and increases, respectively, in relation to the expected frequencies. Only filled tiles represent observed frequencies that were significantly different than expected according to Pearson's chi-square test ( $\mathrm{N}=864$ fruits).

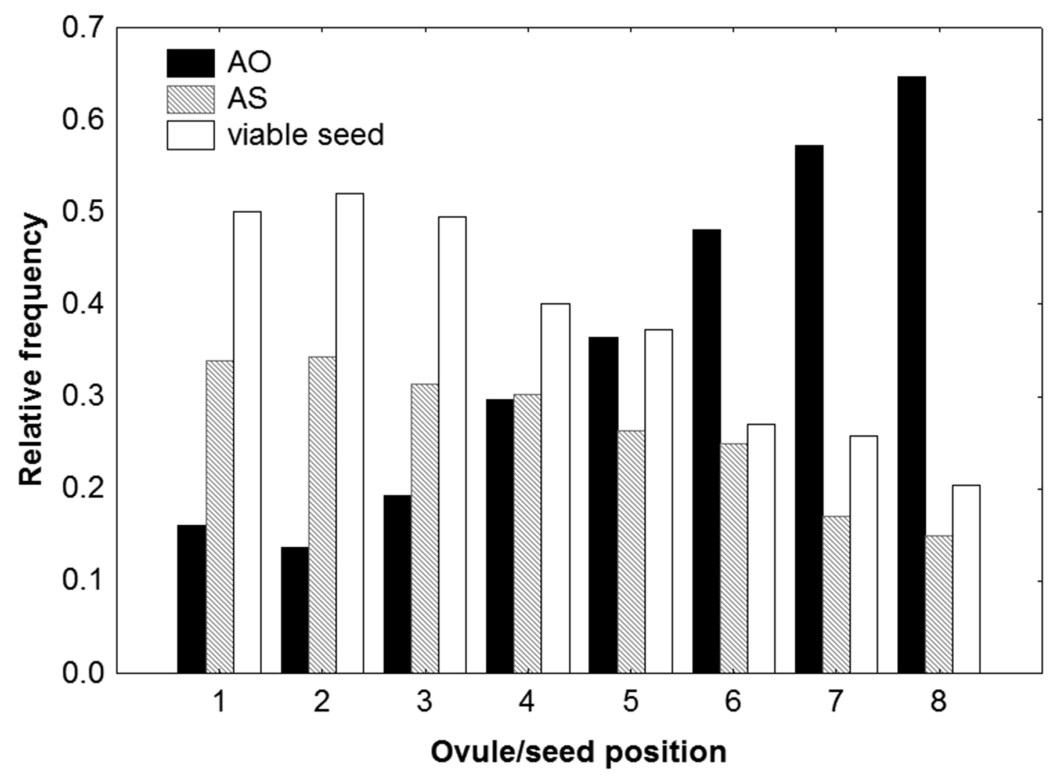

Figure 5. Relative frequency distribution for the number of aborted ovules (AO), aborted seeds (AS) and viable seeds in relation to position within the fruit in Caesalpinia gilliesii. The stylar end corresponds to position 1 .

In addition, ovules of fruits at distal positions within the inflorescence had a higher probability of aborting than did those within fruits at basal or medium positions $(z \geq 2.50$, $p \leq 0.01)$. Fruit position in the inflorescence, however, had no significant effect on the probability that a seed would abort or become viable $(z \leq-1.11, p \geq 0.265$ and $z \leq-1.14$, $p \geq 0.251$, respectively).

The effect of the mother plant (random term) on probability of ovule abortion was significant $\left(\chi_{1}^{2}=106.55, d f=2\right.$, $p<0.0001)$. Similarly, the mother plant had a significant effect on seed abortion probabilities $\left(\chi_{1}^{2}=131.81, d f=3\right.$, $p<0.0001)$ and seed viability $\left(\chi_{1}^{2}=62.48, d f=3, p<0.0001\right)$.
The model variance attributable to the mother plant was $9.38 \%, 11.32 \%$ and $34.96 \%$ for viable seeds, aborted ovules and aborted seeds, respectively. In contrast, the site effect was not significant for any factor: ovule abortion $\left(\chi_{1}^{2}=1.08\right.$, $d f=2, p=0.582)$; seed abortion $\left(\chi_{1}^{2}=4.54, d f=3, p=0.209\right)$; or seed viability $\left(\chi_{1}^{2}=6.625, d f=3, p=0.084\right)$.

\section{Ovule and seed abortion and fruit costs}

The PMS was significantly lower in fruits with an ROA of $100 \%$ than in fruits with lower ROAs $(z \geq-2.88, d f=452$, $p \leq 0.004$; Fig. 6a). However, mean seed mass was similar 
among ROA levels $(z \leq 0.52, d f=452, p \geq 0.598$; Fig. $6 b)$. In addition, the seed number was similar between fruits with an ROA of $0 \%$ and those with an ROA of $100 \%(z=-1.83$, $d f=452, p=0.140$; Fig. $6 c$ ) but was lower in those with intermediate ROAs ( $z \geq-4.89, p \leq 0.0001$; Fig. $6 c)$.

The effect of the mother plant on PMS and mean seed mass was significant $\left(\chi_{1}^{2} \geq 41.08, p<0.0001\right), 21.45 \%$ and $35.15 \%$ of the model variance in PMS and mean seed mass being explained by differences in mother plants, respectively. Nevertheless, the effect of the mother plant on seed number per fruit was negligible $\left(\chi_{1}^{2}=3.08, p=0.079\right)$. Although variability between sites did not have a significant effect on PMS or on mean seed mass per fruit $\left(\chi_{1}^{2}=0.72\right.$, $p \geq 0.395$ ), it did have a significant effect on seed number per fruit $\left(\chi_{1}^{2}=6.12, p=0.013\right)$, explaining $18.01 \%$ of the model variance.

\section{Discussion}

\section{Abortion and brood size}

Both pre-zygotic and post-zygotic ovule abortion (aborted ovules and aborted seeds, respectively) contributed to the brood size observed in Caesalpinia gilliesii, with ovule abortion being more important than seed abortion in one- and two-seeded fruits. The effect of pollination treatment was not significant on the frequency of aborted ovules within the fruit but was significant on aborted seeds, with cross-pollinated fruits having a lower proportion of aborted seeds than open-pollinated fruits. Seed abortion is frequently associated with selection of superior genotypes (Korbecka et al. 2002), which in self-incompatible species depends on pollen quality. Given that C. gilliesii is partially self-incompatible, the association between seed abortion and pollination treatment underscores previous findings about the preference for outcross pollen in this species (Jausoro \& Galetto 2001; Calviño 2006).

Ovule fate was non-random within fruits of Caesalpinia gilliesii with stylar ovules less likely to abort, and with seeds (both aborted and viable) frequently located at stylar positions. This pattern is consistent with that described by Sengupta and Tandon (2010) for several leguminous plants, in which receptive ovules are usually located near the stylar end of the ovary. Although earlier studies in leguminous plants did not usually distinguish between ovule and seed abortion, some showed that stylar ovules set seeds more frequently than do basal ovules in a number leguminous species, a pattern that is related to the site of pollen tube entrance into the ovary (Bawa \& Buckley 1989; Tybrik 1993; Herrera 1999). The prevalence of seeds at positions where ovule abortion is less frequent suggests that seed fertilization and development can take advantage from a pattern of acropetal maturation of ovules (Sengupta \& Tandon 2010).

In addition, the frequencies of aborted ovules were higher in one- and two-seeded fruits of Caesalpinia gil- liesii than in fruits with three or more seeds per fruit. The frequency of aborted ovules in one- and two-seeded fruits was even higher than that of aborted seeds. Thus, considering that one- and two-seeded fruits comprised $34 \%$
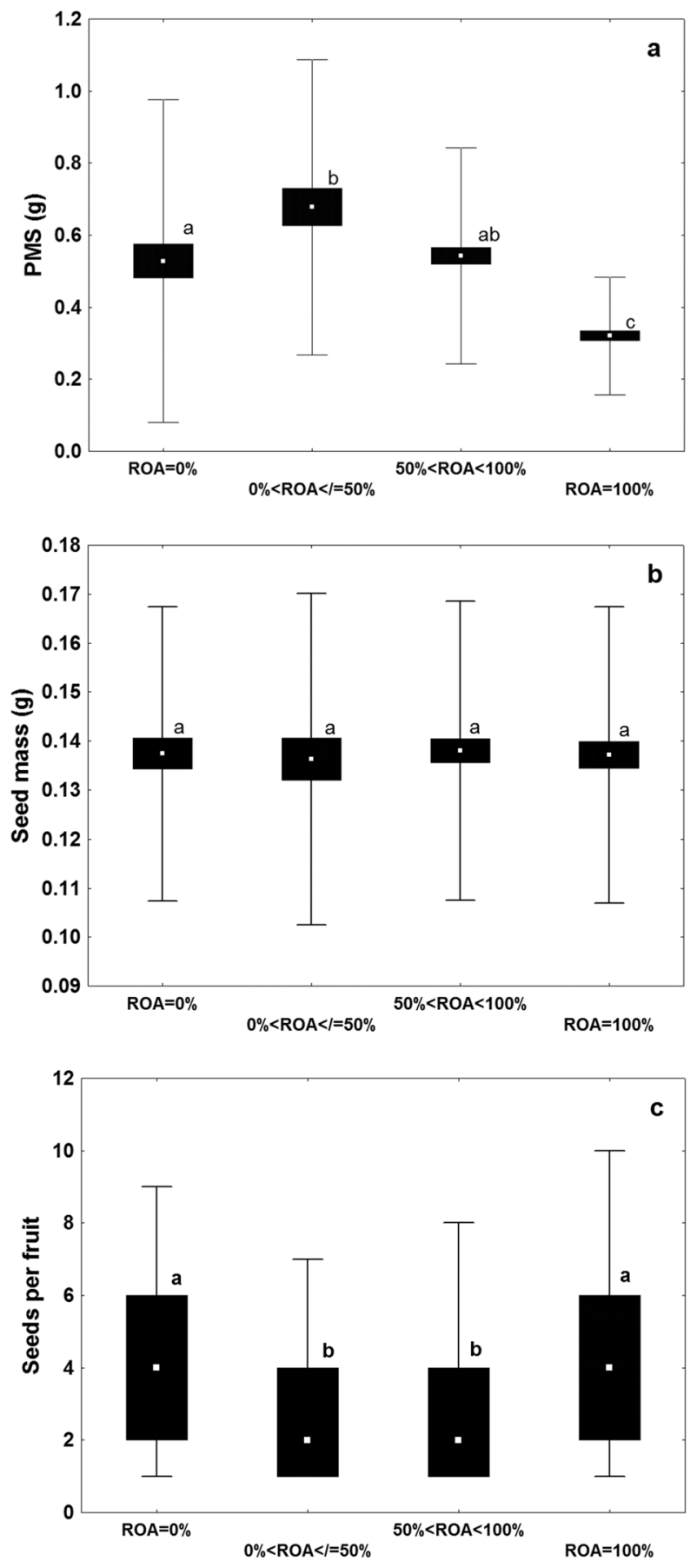

Figure 6. Abortion effects on pericarp mass per seed (PMS, in a), seed mass (in b) and seed number per fruit (in c) in Caesalpinia gilliesii fruits. For each fruit, the relative ovule abortion (ROA) rate was obtained as the proportion of aborted ovules relative to the total number of aborted ovules and seeds. Boxes delimit standard errors (in a and b) and 25-75\% (in c); the white squares within the box are means (in a and b) and medians (in c); whiskers indicate the \pm 1 standard deviations (in a and b) and 90th and 10th percentiles (in c). Different letters indicate statistically significant differences at $p<0.05$. 
of the fruit sample as a whole, the frequency distribution of seed number per fruit would be under a strong influence of ovule abortion in this species. According to Uma Shaanker et al. (1988), positively skewed frequency distributions as the one observed in C. gilliesii may result from a parent-offspring conflict resolved in favor of the offspring. Because maternal cost per seed increases in fruits with low seed number, brood reduction can favor offspring fitness by lowering competition levels between seeds before and after dispersal (Uma Shaanker et al. 1988), as well as, quite probably, between seeds and ovules. Assuming asynchronous ovule maturation, a proximate cause of brood size reduction in C. gilliesii might be simply that seeds in early development are better sinks than are immature ovules, as has been observed in other species (O'Donnel \& Bawa 1993). This condition can lower the chances of plant fruits of increasing seed number even if pollination is enhanced. In fact, the frequency distribution of seed number per fruit in cross- and open-pollinated fruits was similar, showing that cross-pollination fails to increase the frequency of fruits with higher seed number in C. gilliesii. An effect of the quality of the pollen entering the style on seed number, however, cannot be completely discarded. For instance, studies in Narcissus triandrus have shown that pre-zygotic ovule abortion is triggered by the presence of self-pollen tubes (Sage et al. 1999). Accordingly, the higher frequencies of aborted ovules in one- and two-seeded fruits of C. gilliesii observed in the present study might have resulted from selfincompatible pollinations. However, unlike in N. triandrus, we observed post-zygotic ovule abortion in C. gilliesii. The rate of post-zygotic ovule abortion was significantly lower for hand cross-pollinated fruits than for open-pollinated fruits, whereas ovule abortion was similar for both types of pollination treatment. Therefore, in C. gilliesii, pollen quality better accounts for post-zygotic ovule abortion than for pre-zygotic ovule abortion.

\section{Abortion and fruit costs}

Our results show that, in general, higher rates of ovule abortion resulted in lower costs in Caesalpinia gilliesii fruits; fruits that aborted only seeds were more costly than were fruits that aborted only ovules. In addition, the final number of seeds per fruit was similar for all fruits that abort, regardless of whether they abort ovules or seeds. These findings suggest that the difference in cost would lie in the structure that aborts, because fruits that aborted ovules had a similar seed number but lower PMS than did those that aborted seeds. Evidence regarding fruit costs and ovule abortion is scarce; however, studies in Arabidopsis have shown that abortion of ovules prior to fertilization allows the plant to redirect the conserved resources to other floral organs (Sun et al. 2004; Carbonell-Bejerano et al. 2011). It has been shown that seed mass can also significantly increase after induced seed abortion in Cynoglossum officinale (Melser
\& Klinkhamer 2001). For C. gilliesii, however, the average seed mass per fruit was similar regardless of the rate of abortion within the fruit. Therefore, ovule abortion was a more efficient mechanism of reducing fruit costs than was seed abortion, because fruits that aborted ovules reached a similar brood size of similar mass but with a lower PMS than did fruits that aborted seeds. These results support Lloyd's hypothesis that an earlier regulation event (i.e., ovule abortion) is less wasteful for the mother plant (Lloyd 1980) than is a later regulation event (i.e., abortion of developing seeds) because an earlier regulation event incurs in lower fruit costs. Alternatively, if cost of reproduction were expressed in accessory structures other than seed packaging structures, such as stems, peduncles and sterile floral organs (Lord \& Westoby 2006), fruit costs might be underestimated in the present work. However, investment in packaging structures, estimated here as PMS, usually represents the largest contribution to total accessory costs (Lord \& Westoby 2006) and it is therefore unlikely that biomass invested in additional structures would increase accessory costs in C. gilliesii.

Costs associated with fruit production in Caesalpinia gilliesii should also consider the production of aborted, seedless fruits. Because the number of fertilized seeds in seedless fruits was lower than in fruits with one or more seeds per fruit (Calviño, unpublished data), fertilization success within the ovary might have influenced abortion of these fruits. In addition, no seedless (aborted) fruit was obtained in the cross-pollination treatment. However, fruit abortion and brood reduction within the fruit are uncoupled processes (Herrera 1990) and fruit:flower ratios are better explained by mechanisms operating at the whole plant level, whereas seed:ovule ratios are best explained by factors acting at the individual flower level (Holland \& Chamberlain 2007). The cost of producing seedless fruits might therefore depend on the total investment in fruits and seeds made by a plant, whereas for plants producing many fruits, the cost of seedless fruits may dilute among many developing sinks. Accordingly, as total fruit number per plant decreases, the biomass of seedless fruits in $C$. gilliesii increases from less than $1 \%$ to more than $20 \%$ of the total fruit mass (Calviño, unpublished data), suggesting strong maternal control of the production of seedless fruits, operating through whole fruit investment. In fact, seed abortion, which was most common in seedless fruits, was under a stronger maternal influence than was ovule abortion, as evinced by random effects. An effect of the maternal plant on seed abortion and a higher frequency of aborted seeds in open-pollinated fruits suggests an influence of recessive load as the main cause of seed abortion (Kärkkáinen et al. 1999). However, further studies are needed in order to elucidate this effect, as well as to evaluate the environmental (Roach \& Wulff 1987) and genetic (Kärkkáinen et al. 1999) components of the maternal influence on seed fate in C. gilliesii. 


\section{Abortion and fruit position}

In Caesalpinia gilliesii inflorescences, fruit position had a significant effect on ovule abortion but not on seed abortion, reducing the number of fertile ovules available at distal positions within the inflorescence. Because our study was based on a fruit sample, the positional variability attributable to plasticity in fruiting success or architecture alone cannot be distinguished (Diggle 2003). However, ovule number per ovary was similar when bud position was controlled for in fruitless inflorescences (Calviño \& Carrizo García 2005) in favor of fruiting success as the main source of variability in ovule number. In addition, some features indicate that the influence of a predictable pollination environment would be the main source of position-dependent variation in ovule abortion in C. gilliesii. When unpredictability in reproduction or resources is coupled with a reduction in ovule number, positional variability in ovule number can represent a selective advantage (Diggle 2003). Sphingid moths, the specialized pollinators of C. gilliesii (Moré et al. 2006), usually visit flowers of the same inflorescence sequentially (Cocucci et al. 1992), promoting geitonogamous pollination; in agreement with this pollination behavior, fruiting success is lower in the upper third of the inflorescence than in the basal and intermediate positions (Jausoro \& Galetto 2001). Consequently, in this pollination scenario, selecting earlier, less wasteful abortions would seem more reasonable than covering the costs of aborting developing seeds. Reduced ovule numbers at distal positions might therefore have evolved as a strategy to reduce the opportunities of undesired, less successful pollinations.

Most theoretical studies on ovule fate are based on the underlying assumption that aborted ovules were fertilized. However, if pre-zygotic ovule abortion has a significant effect on seed:ovule ratios, as suggested in this and previous works (Sengupta \& Tandon 2010), the determination of the ultimate cause of brood reduction due to pre-zygotic ovule abortion should consider the selective advantage of aborting at the gametophytic stage. The hypothesis raised by Lloyd (1980) clearly indicates an advantage of earlier ovule abortion over later, more wasteful abortion. This advantage is consistent with positional effects on variability in ovule number, provided that the potential of a flower to set fruit becomes predictable at certain positions (Lloyd 1980). However, regardless of positional effects on ovule reduction, a question arises as to whether aborted ovules in Caesalpinia gilliesii are a random sample of the total number of ovules produced by a plant. If, as was found for seed abortion (which is also influenced by positional effects in several species), progeny quality can be improved by a mechanism of pre-zygotic abortion, then selection at the female gametophyte stage can take place. Theoretical evidence supports selection in the female and male gametophytes (Haldane 1924), although empirical evidence is only available for the male gametophyte (Hormaza \& Herrero 1992; but see Koval 2000). Future studies should evaluate the possibility that abortion of the female gametophyte (i.e., ovule abortion) might also be a mechanism for improving sporophyte quality.

\section{Acknowledgments}

The author thanks Mariana de Andrade Iguatemy for the translation of the abstract to Portuguese. This work was supported by grants from the Proyectos de Investigación Científica y Tecnológica (PICT, Scientific and Technological Research Project Fund; Grant no. 2011. 1606) of the Argentine National Agency for the Promotion of Science and Technology, as well as from the Argentine Consejo $\mathrm{Na}$ cional de Investigaciones Científicas y Técnicas (CONICET, National Council for Scientific and Technical Research; Grant no. 00790). In addition, AC is a CONICET researcher.

\section{References}

Alburquerque, N.; Burgos, L. \& Egea, J. 2002. Variability in the developmental stage of apricot ovules at anthesis and its relationship with fruit set. Annals of Applied Biology 141: 147-152.

Bates, D.; Maechler, M. \& Bolker, B. 2011. Ime4: Linear mixed-effects models using S4 classes. R package version 0.999375-42. http:// CRAN.R-project.org/package $=$ lme 4

Bawa, K.S. \& Buckley, B.P. 1989. Seed: ovule ratios, selective seed abortion, and mating system in Leguminosae. Pp. 243-262. In: Stirton, C.H. \& Zarucchi, J.L. (Eds), Advances in legume biology. Monographs of systematic botany of the Missouri Botanical Garden 29. St. Louis, Missouri Botanical Garden.

Calviño, A. \& Carrizo García, C. 2005. Sexual dimorphism and gynoecium size variation in the andromonoecious shrub Caesalpinia gilliesii. Plant Biology 7: 195-202.

Calviño, A. 2006. Evaluación del éxito reproductivo de Caesalpinia gilliesii (Fabaceae) en función de la densidad a distintas escalas. $\mathrm{PhD}$ Dissertation. Universidad Nacional de Córdoba, Cordoba.

Carbonell-Bejerano, P.; Urbez C.; Ganell A.; Carbonell, J. \& Pérez-Amador, M.A. 2011. Ethylene is involved in pistil fate by modulating the onset of ovule senescence and the GA-mediated fruit set in Arabidopsis. BMC Plant Biology 11 : 1-9.

Cocucci, A.A.; Galetto, L. \& Sérsic, A.N. 1992. El síndrome floral de Caesalpinia gilliesii (Fabaceae - Caesalpinioideae). Darwiniana 31: 111-135.

Cuevas, J.; Pinillos, V. \& Polito, V.S. 2009. Effective pollination period in "Manzanillo" and "Picual" olive trees. Journal of Horticultural Science and Biotechnology 84: 370-374.

Diggle, P.K. 2003. Architectural effects on floral form and function: a review. Pp 63-80. In: Stuessy, T., Horandl, E. \& Mayer, V. (Eds.). Deep Morphology: Toward a Renaissance of Morphology in Plant Systematics. Koeltz, Konigstein.

Goldwin, G.K. 1992. Environmental and internal regulation of fruiting with particular reference to Cox's Orange Pippin apple. Pp 75-100. In: Marshall, C. \& Grace, J. (Eds.). Fruit and seed production. Aspects of development, environmental physiology and ecology. Society for experimental biology. Cambridge, Seminar series 47, CUP.

Haldane, J.B.S. 1924. A mathematical theory of natural and artificial selection. Part I. Transactions of the Cambridge Philosophical Society 23: 19-41.

Herrera, C.M. 1990. Brood size reduction in Lavandula latifolia (Labiatae): a test of alternative hypothesis. Evolutionary Trends in Plants 4: 99-105.

Herrera, J. 1999. Fecundity above the species level: ovule number and brood size in the Genisteae (Fabaceae: Papilionoideae). International Journal of Plant Sciences 160: 887-896. 
Holland, J.N. \& Chamberlain, S.A. 2007. Ecological and evolutionary mechanisms for low seed : ovule ratios: need for a pluralistic approach?. Ecology 88: 706-715.

Hormaza, J.I. \& Herrero, M. 1992. Pollen selection. Theoretical Applied Genetics 83: 663-672.

Jausoro, M. \& Galetto, L. 2001. Producción de flores y frutos en una especie andromonoica: Caesalpinia gilliesii. Kurtziana 29: 15-25.

Kärkkáinen, K.; Savolainen, O. \& Koski, V. 1999. Why do plants abort so many developing seeds: bad offspring or bad maternal genotypes? Evolutionary Ecology 13: 305-317.

Korbecka, G.; Klinkhamer, P.G.L. \& Vrieling, K. 2002. Selective embryo abortion hypothesis revisited - a molecular approach. Plant Biology 4: 298-310.

Koval, V.S. 2000. Male and female gametophyte selection of barley for salt tolerance. Hereditas 132: 1-5.

Lloyd, D.G. 1980. Sexual strategies in plants. I. An hypothesis of serial adjustment of maternal investment during one reproductive session. New Phytologist 86: 69-79.

Lord, J.M. \& Westoby, M. 2006. Accessory costs of seed production. Oecologia 150: 310-317.

Melser, C. \& Klinkhamer, P.G.L. .2001. Selective seed abortion increases offspring survival in Cynoglossum officinale (Boraginaceae). American Journal of Botany 88: 1033-1040.

Meyer, D.; Zeileis, A. \& Hornik, K. 2006. The Strucplot Framework: Visualizing Multi-Way Contingency Tables with vcd. Journal of Statistical Software 17: 1-48.

Meyer, D. Zeileis, A. \& Hornik K. 2011. vcd: Visualizing Categorical Data. $\mathrm{R}$ package version 1.2-12.

Moré, M.; Sérsic, A.N. \& Cocucci, A.A. 2006. Specialized use of pollen vectors by Caesalpinia gilliesii, a legume species with brush-type flowers. Biological Journal of the Linnean Society 88: 579-592.

O'Neill, S.D. 1997. Pollination regulation of flower development. Annual Review of Plant Physiology and Plant Molecular Biology 48: 547-574.

O’Donnel, M.E. \& Bawa, K.S. 1993. Gamete selection and pattern of ovule and seed abortion. Current Science 65: 214-219.
Obeso, J.R. 2004. A hierarchical perspective in allocation to reproduction from whole plant to fruit and seed level. Perspectives in Plant Ecology, Evolution and Systematics 6: 217-225.

Pinheiro, J., Bates, D., DebRoy, S., Sarkar, D. \& the R Development Core Team. 2011. nlme: Linear and Nonlinear Mixed Effects Models. $\mathrm{R}$ package version 3.1-102.

R Development Core Team. 2011. R: A language and environment for statistical computing. Vienna, R Foundation for Statistical Computing.

Roach, D.A. \& Wulff, R.D. 1987. Maternal effects in plants. Annual Review of Ecology and Systematics 18: 209-235.

Sage, T.L.; Strumas, F.; Cole, W.W. \& Barrett, S.C.H. 1999. Differential ovule development following self- and cross-pollination: the basis of self-sterility in Narcissus triandrus (Amaryllidaceae). American Journal of Botany 86: 855-870.

Said, C.; Villar, M. \& Zandonella, P. 1991. Ovule receptivity and pollen viability in Japanese larch (Larix leptolepis Gold.). Silvae Genetica 40: 1-6.

Sanzol, J. \& Herrero, M. 2001. The "effective pollination period" in fruit trees. Scientia Horticulturae 90: 1-17.

Sengupta, S. \& Tandon, R. 2010. Assessment of ovule receptivity as a function of expected brood size in flowering plants. International Journal of Plant Reproductive Biology 2: 51-63.

Sun, K.; Hunt, K. \& Hauser, B.A. 2004. Ovule abortion in Arabidopsis triggered by stress. Plant Physiology 135: 2358-2367.

Tybrik, K. 1993. Pollination, breeding system and seed abortion in some African acacias. Botanical Journal of the Linnean Society 112: 107-137.

Uma Shaanker, R.; Ganeshaiah, K.N. \& Bawa, K.S. 1988. Parent-offspring conflict, sibling rivalry, and brood size patterns in plants. Annual Review of Ecology and Systematics 19: 177-205.

Wiens, D.; King, E.J.; Nickrent, D.L.; Calvin, C.L. \& Vivrette, N.L. 1989. Embryo and seed abortion in plants. Nature 342: 625-626.

Zeileis, A.; Meyer, D. \& Hornik, K. 2007. Residual-based shadings for visualizing (conditional) independence. Journal of Computational and Graphical Statistics 16: 507-525.

Zuur, A.F.; Ieno, E.N.; Walker, N.J.; Saveliev, A.A. \& Smith, G.M. 2009. Mixed effects models and extensions in ecology with R. New York, Springer. 